\title{
"All in the Same Boat": Tradeoffs of Voice Assistant Ownership for Mixed-Visual-Ability Families
}

\author{
Kevin M. Storer \\ University of California, Irvine \\ Irvine, CA, USA \\ storerk@uci.edu
}

\author{
Tejinder K. Judge \\ Google Inc. \\ Mountain View, CA, USA \\ tkjudge@google.com
}

\author{
Stacy M. Branham \\ University of California, Irvine \\ Irvine, CA, USA \\ sbranham@uci.edu
}

\begin{abstract}
A growing body of evidence suggests Voice Assistants (VAs) are highly valued by people with vision impairments (PWVI) and much less so by sighted users. Yet, many are deployed in homes where both PWVI and sighted family members reside. Researchers have yet to study whether VA use and perceived benefits are affected in settings where one person has a visual impairment and others do not. We conducted six in-depth interviews with partners to understand patterns of domestic VA use in mixed-visualability families. Although PWVI were more motivated to acquire VAs, used them more frequently, and learned more proactively about their features, partners with vision identified similar benefits and disadvantages of having VAs in their home. We found that the universal usability of VAs both equalizes experience across abilities and presents complex tradeoffs for families - regarding interpersonal relationships, domestic labor, and physical safety-which are weighed against accessibility benefits for PWVI and complicate the decision to fully integrate VAs in the home.
\end{abstract}

\section{Author Keywords}

Voice Assistant; Universal Usability; vision impairment; mixed-visual-ability settings; home.

\section{CSS Concepts}

- Human-centered computing $\sim$ Human computer interaction (HCI) Empirical studies in HCI • Humancentered computing $\sim$ Accessibility $\sim$ Empirical studies in Accessibility

\section{INTRODUCTION}

Voice Assistants (VAs) have become a standard feature of a growing number of digital devices and are used by millions of people around the world [36]. VAs like Google Assistant, Apple's Siri, and Amazon Alexa are available on laptops, mobile phones, and smart speakers to help users perform a range of tasks - from sending text messages to controlling home appliances - using their voices. But, research shows that many users are disappointed in their

Permission to make digital or hard copies of part or all of this work for personal or classroom use is granted without fee provided that copies are not made or distributed for profit or commercial advantage and that copies bear this notice and the full citation on the first page. Copyrights for third-party components of this work must be honored. For all other uses, contact the Owner/Author.

CHI '20, April 25-30, 2020, Honolulu, HI, USA

(C) 2020 Copyright is held by the owner/author(s)

ACM ISBN 978-1-4503-6708-0/20/04.

DOI: https://doi.org/10.1145/3313831.3376225
VA experience [29], and use them less over time, until ultimately abandoning them [16].

Conversely, the value of VAs has been widely recognized by and for people with disabilities (PWD) $[6,17,39,44]$, particularly people with vision impairments (PWVI). The eyes- and hands-free accessibility of VAs has created excitement amongst Accessibility researchers [1,15,40,53] and blind advocacy groups $[26,32]$ alike. For instance, VAs have been noted to make text-entry more efficient $[40,58]$, provide access to inaccessible third-party apps, and reduce stigma in public settings [1].

While people with and without disabilities may have contrasting opinions of VAs, many VAs are deployed in homes where people with and without disabilities live together. Integrating digital technology into the home often involves negotiation and compromise between individual family members [23]. Such negotiations may be particularly complicated when adopting VAs in mixed-visual-ability homes, where family members likely have differing views of VAs' utility as well as different access needs. Technology use in mixed-ability settings ${ }^{1}$ is known to raise tensions $[13,14]$, as many mainstream technologies are not designed to be universally usable ${ }^{2}$ [48]; in other words, they are designed to be accessible to either people with or without disabilities, and access for one user may occlude access for the other. In these situations, PWD have been shown to limit their own access to avoid disrupting nondisabled others $[13,14]$.

Unlike other mainstream technologies, VAs are the first mass-market digital devices whose native, core interactions are non-visual for all users, without need to retrofit accessibility for PWVI [55]. Despite growing interest in how VAs are used by PWD [1,6,17,40,53] and in mixedability settings [33], there have yet to be studies examining whether tensions emerge when adopting and using VAs in mixed-visual-ability households, and how these may affect patterns of VA use, and the roles, relationships, and values in mixed-visual-ability families.

\footnotetext{
${ }^{1}$ Settings where people with different (dis)abilities are present, here, people with and without visual impairments.

${ }^{2}$ We use "universal usability" to indicate users can access VAs regardless of visual ability.
} 
We conducted six semi-structured, remote pair interviews with partners who used VAs in their homes daily for two or more years. We asked partners how they used VAs individually, together, and differently from one another, and to reflect upon whether and how VAs have affected dynamics in the home. We found that, while VAs were universally usable across visual abilities, PWVI were more motivated to acquire them, used them more frequently, and learned more proactively about their features. At the same time, partners with different visual abilities identified similar benefits of having VAs in the home, as well as similar concerns. Broadly, we found that the universal usability of VAs sidesteps the types of direct accessibility conflicts identified in prior work, but also introduces a new set of tradeoffs - regarding interpersonal relationships, domestic labor, and physical safety - which are weighed against accessibility benefits for PWVI and complicate the decision to fully integrate VAs in the home.

\section{RELATED WORK}

Voice Inputs, Audio Outputs, and Non-Visual Access

While conversational interaction is unique to VAs, audioout and voice-in modalities have long been used to provide access to PWVI in a variety of other digital tools. For example, auditory outputs are leveraged in screen readers, like JAWS [62] and NVDA [63], as well as "talking" technologies, like clocks and calculators [64]. In HumanComputer Interaction (HCI) and AT research, audio outputs for PWVI have been used in a variety of domains, like accessible web traversal [11,12], graph translation [2], and wayfinding $[22,43,58]$. More recently, the turn to inaccessible or unusable touchscreens [25] and the increased fidelity of speech recognition technologies have prompted explorations into the use of voice inputs by PWVI. For example, Azenkot and Lee found that PWVI use voice inputs on mobile phones more frequently than sighted users, primarily because it is more efficient than keyboard text entry [5]. Ye et al. also found PWVI are more likely to use voice inputs on their phones to enter text because of their efficiency, despite raising concerns in public and work settings [60].

\section{Voice Assistants and People with Vision Impairments}

The widespread availability of VAs like Google Assistant, Cortana, Siri, and Alexa, has been a topic of recent interest in HCI $[4,8,10,21,27,28,34,35,38,41,59]$. Many have begun inquiries into the potential of child-VA interactiondocumenting the types of interactions children seek $[21,28]$, how they form questions [27,35,59], and opportunities for social play [35]. Regarding use by adults and families in the home, research has tended to focus on detailed conversational patterns $[8,38]$, critiquing the degree to which VAs are in fact conversational and identifying ways to support more efficient repair of breakdowns. While there have been studies of broader patterns of use by families, these have relied heavily on $\log$ data $[4,10,45]$. Key conclusions include: median usage (between 4-10 commands per day), most used features (media, hands-free search, setting timers, controlling lights, etc.), and presence of privacy concerns (fear of companies sharing or selling voice data). So, although our study is one of mixed-visualability families, it is one of the first to answer Ammari et al.'s call for future work to "document family routines" and parenting behaviors of "more varied pools of users" [4].

Despite reports of regular daily VA use [4,10], several studies report on the limitations of VAs for people who are sighted $[16,18,29]$. Cowan et al. document a range of frustrations of "infrequent users" of VAs [18]. Luger and Sellen found that users expect VAs to be more useful or intelligent than they are in practice, and ultimately express disappointment with their VA experience [29]. Similarly, in a longitudinal deployment, Cho et al. found that use stagnates and declines over time as users come to accept the limitations of their device [16]. By contrast, many studies of VA use by people with disabilities (PWD), for example people with motor [17], cognitive [6], and age-related $[39,44]$ impairments, report generally positive experiences, suggesting VAs are a universally usable technology [48]. Likewise, when examining a range of disabilities, Pradhan et al. found that some $85 \%$ of Amazon Alexa reviews related to disability were positive [40].

Because their core interaction mechanisms are non-visual, a growing body of work has explored VA use specifically by PWVI; findings suggest that PWVI also generally perceive VAs positively. For example, following a content analysis of product reviews, Pradhan et al. interviewed PWVI and found that many PWVI perceived VAs as "an integral part of their lives" [40]. Similarly, Abdolrahmani et al. found that VAs contributed to feelings of "empowerment" among PWVI [1]. Vtyurina et al. even found that PWVI identified some advantages of VAs over traditional AT like screen readers, particularly that they are easier to learn [53].

This divergence in experiences between PWVI and sighted users has prompted some of these scholars to suggest there may be fundamental differences in the patterns of use of VAs across visual abilities. For instance, Pradhan et al. [40] state that their finding regarding PWVI contrasts the conclusion drawn by Luger and Sellen [29] that sighted users perceive VAs to be "gimmicky"-a difference that may be attributable to either differences in devices or abilities. Similarly, Abdolrahmani et al. [1] suggest that PWVI may use VAs for fundamentally different types of tasks than sighted users, like work-related tasks. Further, PWVI may be more willing to use imperfect VAs because they have no other alternative to access certain third-party apps [1]. Perhaps this lack of alternatives allows PWVI to remain optimistic about the current capabilities and future potential of VAs, despite a lack of customizability in the interface (like variability of the voice speed) which would allow for even more efficient interactions [15]. The present study expands on these works by examining underlying explanations of this apparent dichotomy in VA experience across visual abilities. 


\section{Mixed-Ability Settings}

The ability to complete daily tasks independently has long been a fundamental goal of AT design, though recent work has noted the collaborative and interdependent nature of constructing access [9,51], often in mixed-ability settings.

Several works have explored the potential of universally usable interfaces to support collaborations between people with and without disabilities. Winberg and Bowers developed a digital "Towers of Hanoi" game with parallel visual and audio outputs for mixed-visual-ability teams [57]. Piper and Hollan designed a digital tabletop display with speech-to-text functionality to support communication between hearing care-providers and Deaf care-recipients [37]. Thieme et al. designed Torino, a tangible, audio-based programming language to facilitate mixed-visual-ability pair programming for children [52]. Metatla et al. explored the potential for universally usable VAs to support inclusive learning for young students with and without visual impairments in co-design workshops [33].

One challenge of mixed-ability collaboration is that perspectives of people with and without disabilities can conflict with individual access needs. Branham and Kane found that the views of accessibility held by employers, who often provide only legally-required accommodations, can put the impetus on PWVI to create access for themselves when faced with ineffective policies [14]. Williams et al. found that PWVI and sighted walking partners have fundamentally different views of wayfinding, such that sighted partners' well-intended guidance may make navigating more difficult for PWVI [56]. Shinohara et al. found that people with and without disabilities may also have divergent perspectives on digital technology designs, including non-functional aspects, like social propriety [46].

When access needs are not being met, people with varying abilities often negotiate and create access together. Wang and Piper found that successful collaborations between Deaf and hearing coworkers often involve continuously negotiating access by flexibly switching communication mediums [54]. Branham and Kane found that mixed-visualability housemates collaboratively co-construct access through planning, preparation, and intervention activities [13]. Yuan et al. found that PWVI and sighted companions fill gaps in information available to each other when grocery shopping, by establishing common ground [61]. Thieme et al. identified ways that PWVI of varying acuities negotiate access for themselves and each other by sharing and triangulating distributed sensory information [51]. Similarly, Storer and Branham found that blind parents and their pre-literate children use their respective abilities to spell and to see, to collaboratively read textual content that is unintelligible to each individually [49]. We contribute to this body of work by exploring how VAs - mainstream, universally usable technologies - are used in mixed-visualability families and the ways in which they mediate interactions and domestic labor in the home.

\section{Domestic Technology and Familial Considerations}

Studying technology in domestic contexts often requires a deeply reflective research stance [7], in order to surface the ways technology use and adoption are affected by the complex connections between interpersonal dynamics, social roles, and domestic labor in families. Familial considerations can complicate the decision to integrate and use technologies in homes, because adoption may require agreement between multiple parties with different priorities [23]. Alternatively, familial considerations can be a catalyst for adopting technologies. For example, Judge et al. found that the importance of maintaining familial relationships can prompt users to deprioritize concerns about using a technology, like loss of privacy, which are significant barriers to use in other contexts [24].

After technologies are adopted into the home, they can have nuanced and unanticipated impacts on family life. For example, Mazmanian and Lanette found integrating new technologies in homes can create new responsibilities for occupying social roles within a family [31]. Additionally, Rode found that the "digital housekeeping" involved in administrating shared devices often disproportionately complicates social roles for women, where demonstrating technical self-efficacy may increase gender equity in their families' interpersonal dynamics, but also add to their domestic labor [42]. Ames et al. found that digital tools intended to maintain familial relationships, like videoconferencing systems, may also require substantial domestic labor to use [3]. Conversely, Thayer et al. found that digital tools intended to decrease domestic labor, like shared calendars, may also require family members to renegotiate their interpersonal dynamics [50].

Though less is known about the role of AT within domestic contexts, Dawe found that the decision to adopt AT involves agreement between multiple family members with different priorities [19], and Branham and Kane found inaccessibility affects interpersonal dynamics, social roles, and domestic labor in mixed-visual-ability homes [13], similarly to the above works examining families without specified disabilities. We expand on this work by exploring how access created by VAs impacts, and is impacted by, these complex factors.

\section{INTERVIEWS}

\section{Participants}

For our interviews, we recruited six mixed-visual-ability intimate couples through an existing list of interested participants and national blind advocacy groups, over a period of eight weeks. To be included in this study, participant pairs 1) self-identified as cohabiting intimate partners, where 2) only one used a screen reader as their primary mode of accessing digital technologies, who 3) had at least one child 5-13 years old, and 4) owned a smartspeaker VA. There were no other inclusion criteria. Each partner received $\$ 100$ for their time. 


\begin{tabular}{|c|c|c|c|c|c|c|}
\hline Partner 1 & Partner 2 & P1 Visual Ability & P1 AT (in addition to Screen Reader) & Children & VAs Owned $^{2}$ & Other Disabilities \\
\hline Vince & Paula & Light Perception & $\begin{array}{l}\text { Screen Magnification, High-Contrast } \\
\text { Displays, Keyboard-Only } \\
\text { Navigation, White Cane }\end{array}$ & 2 , Ages $8 \& 16$ & GA, AS & $\begin{array}{l}\text { Paula is legally blind with } \\
\text { acuity of } \sim 20 / 200 \text {, uses } \\
\text { vision for many tasks, and } \\
\text { screen magnification tools }\end{array}$ \\
\hline Ben & Carrie & Completely Blind & $\begin{array}{l}\text { Braille Terminal, Keyboard-Only } \\
\text { Navigation, White Cane }\end{array}$ & 2 , Ages $2 \& 9$ & GA, AA & $\begin{array}{l}\text { Carrie is hard of hearing } \\
\text { with 50\% acuity, uses } \\
\text { hearing aids, captions, and } \\
\text { amplifying headphones }\end{array}$ \\
\hline Fatima & Ahmed & Light Perception & Braille Terminal, White Cane & 1, Age 7 & $\mathrm{GA}, \mathrm{AS}, \mathrm{AA}$ & None \\
\hline Jen & Daniel & Acuity $\sim 20 / 500$ & $\begin{array}{l}\text { Screen Magnification, Bioptics } \\
\text { (high-magnification eyeglasses), } \\
\text { Guide Dog }\end{array}$ & 1, Age 12 & GA, AS & $\begin{array}{l}\text { Daughter has visual acuity } \\
\text { of } \sim 20 / 150 \text {, uses screen } \\
\text { reader situationally }\end{array}$ \\
\hline Ryan & Cooper & Acuity $\sim 20 / 200$ & $\begin{array}{l}\text { Screen Magnification, High-Contrast } \\
\text { Displays, Alternative Input Devices }\end{array}$ & 5, Ages N/A & GA, AS, AA & None \\
\hline Hung & Yvette & Completely Blind & $\begin{array}{l}\text { Braille Terminal, Keyboard-Only } \\
\text { Navigation, White Cane }\end{array}$ & 2, Ages $6 \& 8$ & GA, AS & None \\
\hline
\end{tabular}

Table 1: Summary of participant demographics. All participants pseudonymized.

Each pair owned a smart speaker for over two years and used VAs multiple times per day.

${ }^{1}$ Ryan and Cooper declined to report the ages of their children.

${ }^{2}$ VAs Owned abbreviated as Google Assistant (GA), Apple Siri (AS), and Amazon Alexa (AA)

Interviewees ranged in reported age from 30-50+. Four pairs had more than one child. Children living in the household ranged in age from 2-16, with one family declining to report their children's exact ages. Children were not interviewed, however parents often discussed VA interactions that included them. In three pairs, a family member other than the identified screen reader user (Partner 1 in Table 1), also identified as having a disability. In one interviewed pair, Partner 2 identified as legally blind, but accessed digital technologies using vision and screen magnification. In another, Partner 2 identified as hard of hearing, and used hearing aids, closed captioning, live transcription, and sound amplifying headphones. Finally, in a third family, the only child had a vision impairment (reported visual acuity of 20/150), and used a screen reader situationally. The interviewed screen reader users used a variety of other AT, including white canes, braille terminals, and alternative input devices. A full list of reported visual abilities and AT use of the interviewees and their families can be found in Table 1 .

\section{Procedure}

We conducted interviews remotely using Google Meet. This allowed us to maximize the reach of our study, and increase the geographic diversity of our sample. Each remote meeting lasted approximately 90 minutes, and included an introduction to the study, the interview session, and a debriefing period. The content of each interview session was video- and audio- recorded. Interviews lasted between 50 and 94 minutes, averaging 80 minutes per pair. In total, we collected 8 hours of data from six pairs.

Broadly, we asked participants 1) how they use VAs individually, 2) how they use VAs with other family members, 3 ) whether and how they performed the identified tasks prior to owning a VA, 4) what performing the identified tasks with a VA has changed for them, 5) what they would like to be able to do with VAs that they cannot currently, and 6) why a VA would be better than their current strategy. Participants' answers and follow-up questions often explored tradeoffs involved in owning VAs.

Our interview script was structured using the example of Google Assistant as the VA in question. However, each pair owned at least one other VA, including Apple's Siri and/or Amazon's Alexa. Participants often did not distinguish between VAs, and frequently employed comparisons between VAs or stories about using a variety of VAs in their responses. As such, we note that the insights of our participants are not particular to Google Assistant, nor are our findings specific to Google Assistant.

\section{Analysis}

The first author conducted all of the interviews and took detailed notes during each session. All audio and video recordings were transcribed in full, using automated software. From these transcriptions and typed notes, we developed open codes to capture commonalities between participants' responses. We grouped open codes which were common to multiple participant pairs into wider axial codes. Each axial code reported in our analysis was common to at least four of six participant pairs. After all authors agreed to the validity of the axial codes identified, the first author returned to the raw video recordings and transcribed quoted passages manually. In reporting our findings, we denote participants who are not screen reader users with $(\mathrm{V})$, to mean that they use vision to navigate digital devices, including Paula, who is legally blind. 


\section{FINDINGS}

\section{Comparing VA Use Between PWVI and their Partners}

At a high level, we observed differences in domestic VA use between PWVI and their partners. We found that PWVI were more often the first person interested in acquiring a VA, used VAs more frequently, and were more proactive in learning about VAs' functions than their partners. Interestingly, the types of tasks which were performed were more similar between partners in the same home, than they were between partners with the same visual abilities.

In five pairs PWVI were the household member who was first interested in acquiring an in-home VA, and in the sixth pair, both partners were interested. Jen and Daniel's (V) daughter, who has a vision impairment, first brought VAs into their home when she asked for a smart-speaker as a Christmas gift. Carrie (V) shared that she did not know that Ben had even ordered a smart-speaker VA, until the shipping box had arrived. Even for Vince and Paula (V), who both have vision impairments, Paula was not as excited about acquiring a VA as Vince, and thought it would be "basically for Googling things with your voice."

Likewise, PWVI used VAs in the home more frequently than their partners. In each of the interviewed pairs, the participant who was a screen reader user reported using VAs more often than, or equally as much as, their partner. In Jen and Daniel's (V) home, VA use corresponded directly to visual acuity: Jen was the most frequent user, followed by their daughter who has more vision than Jen, while Daniel (V) used VAs least often. In some cases, like Hung and Yvette's (V), sighted partners expressed using VAs little, or not at all. Yvette (V) shared:

$Y(V)$ : I still don't [use the VA]. You know, I see it as something that Hung and the kids do - and I think it's fun that they do, and I'm glad they do, and it's useful. But, I don't do... (interrupts herself to ask Hung) What do I use it for?

H: ...I encourage her to use it for timers, that sort of thing. But, it's still, it's still unnatural, I guess, for her.

Additionally, PWVI learned about the features of their VAs more proactively than their partners. PWVI frequently sought information about VAs online, on technology review sites, or in technology-affinity groups. By contrast, their partners primarily learned to use VAs from the moreknowledgeable PWVI. For example, Paula (V) often learned about features of their VAs from Vince who searched for this information online. Similarly, Yvette (V) learned how to use their VA by "trying to copy" Hung, who learned about their features through his social media connections. Ben, similarly, learned about the features of VAs from social media, while Carrie (V) learned through brute-force-trial-and-error or from Ben. They shared:

B: The 'Eyes-Free List' is a Google Group... I get it in 'digest mode' in my email... [They share things] like, for example the Assistant can now turn on and off TalkBack, just like Siri can turn on and off VoiceOver. That's very recent...

$C(V)$ : If I'm thinking about something, I'll just ask and 'you know who' [the VA wakeword] pops up. And it's like 'Oh, hey! It can do this!' But, usually, I find out more stuff from Ben... He'll be like, 'Hey, babe! You got to check this out!' [or] have you tried this feature out?' And I'm like, 'No. I didn't know it could do that!'

Although we observed differences in the motivation to acquire, use, and learn about VAs across visual abilities, partners mutually identified eyes-free, hands-free accessibility as the primary purpose of using VAs instead of other methods. But, the way they expressed this value was different, where PWVI recognized the role of vision and sighted participants did not. That is, sighted participants primarily described scenarios where VAs were appropriate-those where they had situational visual impairments (e.g., while cooking, or to multitask while looking at another screened device). By contrast, we found that PWVI often described the value of VAs in relation to an inaccessible method for completing the same task, in particular, setting timers, controlling home environments, and shopping. For example, when asked about the value of VAs timers, Hung concisely stated he does not use the stove's timers, because "that's not accessible." Though Paula (V) could set timers on the microwave, when asked why the family now used VAs' timers, Vince interjected:

$V$ : Because vision! Because of vision, it just takes so much more effort... when you could just bark orders at our robot friend, over here.

Similarly, PWVI often compared using VAs to control home environments against the inaccessibility of traditional home infrastructure, especially thermostats. Though her family did not own a VA-connected thermostat, Fatima was interested in purchasing one, and explained:

$F:$ I do want to get the thermostat, definitely, because it will give me [an alternative to] figuring out, 'Okay, what's the temperature?' ... [Now,] I just randomly press buttons, and I don't know what I'm doing.

PWVI also often indicated the value of VAs in contrast to accessibility barriers in routine household shopping. For example, even though Ryan and Cooper (V), both frequently ordered household items through their VA, visual abilities affected when they placed orders, because they considered the cost of accessible methods for getting to the store. While they agreed that they preferred not to ship orders of single items, Ryan was willing to purchase a single item "if [they] really need something," because the cost of shipping that item may be less than the cost of the rideshare he would take to make the purchase in person.

Despite differences in frequency of VA use, and vision playing a primary role in VA use for both partners, we 
found virtually no difference in the types of tasks performed by PWVI and their partners. Many tasks, like timers and information retrieval, were commonly performed by all participants. Less common tasks, however, were primarily performed by both or neither partner in a household. For example, in five of six pairs, neither partner made purchases on their VAs, because partners often had agreements about whether to trust VAs with shared credit card information. Ryan and Cooper (V) were the only pair who agreed to trust VAs - and only certain VAs - to place orders, because of the accessibility benefits for Ryan. But, both indicated making purchases often and independently added needed items to a list stored in a shared virtual shopping-cart. So, while some tasks were prompted by accessibility, they often became common to both partners.

\section{Tradeoffs of Adopting Voice Assistants}

Although partners across visual abilities identified eyesfree, hands-free accessibility as the primary value of VAs, they described a wide variety of social considerations outside of accessibility in their assessments of VAs. Participants routinely identified VAs' potential impacts on 1) interpersonal relationships, 2) domestic labor, and 3) physical safety as major considerations of owning and using VAs. In each domain, VAs presented tradeoffs that were weighed against each other, and against accessibility.

\section{Interpersonal Relationships}

In assessing their overall VA experiences, partners described numerous positive impacts of owning VAs on the interpersonal relationships in their homes. Many families used VAs to play games together, because traditional board and card games are inaccessible to PWVI. Ben and Carrie (V) particularly enjoyed playing audio adventure games with their children, because "that [VA] girl, is gutsy, man! She's not afraid of anything [in the fictional dungeon]." While the voice-only medium of a VA might seem restrictive, providing a limited or subpar gaming experience, we saw similar enthusiasm across all families. VAs games were as fun as, if not more fun than, games in other formats, in part because voice allowed all family members to more frequently play together. Jen found VAs made it easier for her to be included in family games:

$J$ : It's hard for me to see the board game, or read the cards... So, I find myself saying, like, 'No, fine. You guys go play a board game [without me]'... I can play the games more now, because you don't need any cards.

Yvette (V) even suggested VAs created a more equal play experience for her, Hung, and their children. She shared:

Y: Print does get in the way of our games, sometimes... [and VA trivia] kind of equalizes our experience, because we're all in the same boat, hearing the question together.

Daniel (V) and his daughter, who has a vision impairment, played games together nightly, to pass the time while Daniel administered her prescription eyedrops:
$D(V)$ : At night, I have to give her four eyedrops. But, they have to be spaced out. So, I sit with her, and then, just to kill time, we ask [the VA] to play a game... And, you know, either you sit there, just staring at the wall, or we play a game [together].

Some families made up their own games to play using VAs. Jen and Daniel's (V) family played the " $0 / 0$ Game" in which family members took turns asking the VA to divide zero by zero, and the person who received a specific response won. Hung and his kids played together by asking their VA things like "what does a random, strange animal sound like?" and testing "the breadth of knowledge that [the VA] is capable of - or incapable of - giving responses to."

In addition to playing games together, several families used VAs as in-home intercom systems to speak to each other more easily. Some families used this feature primarily to call children from their rooms for dinner or to leave for school. But, Vince and his son used this feature to tell each other jokes, while on separate floors of their house. In other examples, simply learning how to use VAs was a bonding experience that allowed everyone in the house, regardless of visual ability or age, to participate. For instance, Vince and Paula's (V) family acquired their first VA on Christmas, and Vince recounted how they spent the evening exploring the VA's potential together with extended family:

$V$ : We're bored, and there we are sitting around barking orders at [the VA]... sitting around it for, like, four hours or something, all of us and the kids, and they're asking it jokes. It was just funny because every individual in the house [was there, and] it was really different what everyone was asking. You know, you can ask it to make fart sounds - and it'll do it.

Many interpersonal experiences occurring with and around VAs were positive. But, several families identified ways that integrating VAs into their homes had created interpersonal tensions. For example, Hung and Yvette (V) initially had difficulty agreeing to purchase a VA for their home, because she was concerned digital devices might displace interpersonal connections with their two children:

$Y(V)$ : When they were younger, I would try not to have my phone open... There was a time, I told [Hung], 'I don't want the computer voice in the living room...' [Because] they weren't old enough to really get, for me, to get boundaries... [Talking about VAs,] I'm kind of wondering right now, 'How did they get in the house?'

Ryan and Cooper (V), frequently struggled to share VAs with their sons, because, as Ryan explained, one of them "uses it for bull****. He'll ask about new Twitch tournaments and stuff, and I'm like 'Get the hell off! I'm trying to do something." Cooper (V) and the children also used VAs during debates, to fact-check Ryan, who is "gonna sulk about it," when he is wrong. In a more extreme example, they explained that providing VAs with their credit card information to make household purchases also 
allowed their adult son to make unsanctioned purchases. Ryan recounted his son's purchase of a new MacBook:

$R$ : I had no intention on buying him a new [freaking] MacBook... the next thing I know is-it's comin'. And I'm like, 'Okay, who the [hell] ordered a MacBook?'... That [ticked] me off, because I felt that that was stealing from me...

Despite the anger and trust issues that ensued, Cooper (V) and Ryan decided to keep the credit card connected to the account to support Ryan's access to grocery shopping. This purchase was perhaps the most extreme example, but most pairs had at least one example of how using VAs caused headaches for other family members. Daniel (V) and his daughter have "freaked Jen out" using VAs as intercoms to communicate, and startling her when she thought she was alone. Similarly, Vince and Paula (V) had unintentionally awoken family members by using VAs as intercoms.

\section{Domestic Labor}

Participants often pointed to ways VAs had eased the burden of domestic labor in their homes, when assessing their experience. Setting timers, finding recipes, and helping their children with homework were frequent uses of VAs for domestic labor for our participants. In addition to using timers for tasks like cooking and monitoring laundry cycles, our participants often used timers as part of their parenting. Hung and Yvette (V) used VAs to set timers for their children's timeouts. Ben and Carrie (V) used VAs' timers to let their son know when it was time to start homework, or he had reached his daily quota of "screen time." Conversely, Vince used timers to monitor his own media consumption, to remember to put his kids in bed:

$V$ : I play video games, sometimes... [And] the kids used to pull, you know, they're like, 'Dad's going to forget to put me in bed and brush my teeth'... with the timer, it's totally more structured.

Using VAs to find recipes was also common for our participants, although some, like Hung and Yvette (V) removed their VAs from the kitchen to avoid getting food on them. Fatima frequently used VAs to search for recipes, and noted that other digital recipes have trended further toward prioritizing visual content, in recent years:

F: If I'm looking for how to, like, make a cake... I usually just ask [whatever VA] is in front of me, at that time, and ask for the recipe... Before, [I would] go to my laptop, go to the website, go to Google, type what I'm looking for, 'chocolate cake' or whatever, and then open it up. Nowadays, the annoying thing is everything is on YouTube. So, it is very hard for me to find a text version of a recipe written down... That doesn't help me at all. They're showing more, playing music, and talking very little -I have no clue what they're doing.

Four of our six pairs identified VAs as a central part of helping with their children's homework. The remaining two had children who were either too young (Fatima and Ahmed (V)) or too old (Ryan and Cooper (V)) to seek homework help. Hung and Yvette (V) also used VAs to answer questions that had arisen for their children during the school day and encouraged their children to ask the VA how to spell words, because "there's only so many times that somebody can repeat how to spell a word before it gets frustrating." Carrie (V) used VAs to teach herself Common Core pedagogy, to help with her son's math homework. Vince and Paula (V) used VAs to help with their eldest daughter's homework, which covered unfamiliar topics:

$V$ : [The VA] will highlight the basics to really get them started-and us. So, we can better teach our kids. We can, kind of, confirm, because we're a little rusty on some of that stuff.

$P(V)$ : Before we got the [VA], it was just getting on the computer... For him, it takes him a little bit longer to navigate through certain websites. Me, having vision, I can see search results, right in front of me, and I can click on what is most relevant.

Interestingly, in one case, VAs prompted new domestic contributions by providing access to tasks which were tangential to completing the chore. Before Vince and Paula (V) had VAs, Paula was responsible for the family's laundry. But, VAs prompted Vince to do laundry, too:

V: You know why I actually do laundry? It's because I can stream Twitch with [the VA on my TV] ... So, while I'm doing all this folding and hanging, I'm able to turn my TV on in my room. I'm able to alter the volume. I'm able to tell it to go directly to my favorite YouTube channel and play the latest videos, or tell it to play the latest podcasts. So, I think it eases the pain...

$P(V)$ : That's taken a little [work] off me, for sure.

While VAs assisted in performing domestic labor, many participants also identified new household responsibilities which were involved in owning VAs. Hung and Yvette (V), for example, had concerns about the access to digital content afforded to their children by VAs' non-textual interactions. To ensure that their children did not encounter inappropriate content, Hung felt it was important to be physically present each time they used the VA. They were also concerned about how the feminine representation of many VAs and the ability to make requests of VAs without manners might impact their children's respect for humans:

H: The part that's really challenging for our familyand others-is the concept of giving a request to a machine without the whole concept of respect and politeness...

Y: And they're starting to get to the age where they think it's funny not to be nice... That's a big part of [why I resisted using VAs] when they were younger. I didn't want them to get into a loop of playing with a machine and reflecting back the worst of themselves. 
H: I think another concern that I've had more recently is that the default voice of [VAs] is female... There's all kinds of submission/submissive issues, all kinds of stereotypical challenges around the narrative of giving requests to a gendered assistant. I don't necessarily have the language, at the moment, to discuss what it is that I'm trying to get them to understand, other than the fact that it's really important that we give our requests respectfully - to whatever medium.

Similarly, because Vince uses VAs to help his children with their homework, he also had to teach their son about the importance of learning when he asked, "Why do we need to know math, if I can just use [the VA]?" He and Paula (V) ultimately instituted a policy of using VAs only to check answers. Jen and Daniel (V) had instituted a similar policy for limiting their daughter's VA use for schoolwork.

The new responsibilities created by VAs for Ryan and Cooper (V) were quite different from these examples; after their son's purchase of the MacBook, described above, they looked for ways to limit their children's transactions, or to be notified when their children attempted purchases. But, at the time of our interview, they had not found a solution.

Although the added labor of attending to the responsibilities created by VAs was often mitigated by the reduced burden of labor eased by access in other domestic tasks, when examined together, it is difficult to conclude whether VAs decrease or increase domestic labor for families. Notably, the introduction of new domestic labor due to VAs in the home was identified by the majority of households, though our questions did not inquire about burdens of ownership.

\section{Physical Safety}

Many participants identified ways that VAs could increase the safety of themselves and their families. Only three pairs owned VA-connected smart-home devices. But they and two pairs without home automation devices each identified their potential to increase household safety by providing access for PWVI. For example, Hung used his VA to turn the lights on for his family, because "as a blind person, I can't tell when the lights are on. It's kind of nice to get some verbal confirmation." Vince used VAs to turn off his TV, and noted that this was an improvement over "knocking it off the stand, in a hustle to go catch the bus," which had happened to him in the past. But the risk of injury of stumbling in the dark or falling TV sets may be relatively small, when compared to the benefits of other smart-home technologies. For example, Jen was interested in purchasing a smart doorbell, and explained:

$J$ : It would be great if it announced to me, 'Hey, so-andso is at the door,' because I don't see who's at the door... My dad is blind, and for him, it would be a safety feature to know who is at the door, because [he] does not open up the door for anybody. [So], we do have a code [ring pattern], so he [knows] it's family.
Similarly, Fatima explained that being able to use VAs to automate her home would help her to remain safe while cooking. She recounted a time her house nearly caught fire because of her current oven's inaccessibility:

F: I was cooking, and my oven has no Braille, no raised [indicators], no buttons... I probably put it up too high. I don't know, because I can't tell-it's touchscreen. I couldn't control it, and then it started, like, burning my eyes. I had no idea what was happening... I had to find a neighbor to come help me turn the oven off... When [Ahmed] came home, he saw smoke everywhere.

Though participants frequently identified ways VAs' accessibility for PWVI could support safety for the whole family, many weighed this against perceived threats of integrating VAs into their home. Most often participants' fears about using VAs were due to the threat of malicious actors. The potential for strangers to "listen in" on the home through the VA was discussed by the majority of participants. Though Ryan called him "paranoid," Cooper (V) viewed privacy infringement as a threat to safety:

$C(V)$ : Nowadays, the government is getting hacked. So, who's to say that some, you know, thief or mastermind is not able to access [our] information and see my location or see what I'm doing, so they can rob me or something of that nature? That's what it comes down to.

R: Yeah, but all they're gonna get is 'Hey, I want some almond milk.' (laughs)

Other participants expressed similar concerns about the safety implications of using home automation devices, despite the frequent recognition they would increase accessibility for PWVI and, consequently, the household's safety. Ben and Carrie (V) did not own home automation technologies, because they worried "some nut-job" might be able to unlock their door, or frighten their children by turning on their lights during the night. Ahmed (V) expressed similar concerns about to potential for malicious actors to tamper with their access to their home. He shared:

$A(V)$ : I'm not a fan of [smart home automation], because there's hackers out here... I had a friend of mine - he had everything [connected]. Now, he's got a million-dollar home [that] he can't get in.

Though perceived threats were sometimes rooted in hearsay or misinformation, they were often salient enough for participants to alter their use of VAs, or to forgo acquiring VA-connected devices, altogether.

\section{DISCUSSION}

\section{Patterns of VA Use in Mixed-Visual-Ability Families}

Prior work has noted that PWVI and sighted users have seemingly-contrasting views on the value of VAs, which may be attributable to differences in user preferences [40] or in the types of tasks they perform with VAs [1]. But, in our sample, partners with and without visual impairments 
had similar preferences for interacting with VAs and used VAs for similar types of tasks.

Partners with and without visual impairments both identified eyes- and hands-free interactions as the primary value of using VAs. Prior work found sighted users become frustrated when they need to employ their hands in VA interactions [18]. But, our findings suggest sighted users also become frustrated when they need to employ their eyes to use VAs. This contradicts some previous VA design recommendations, which suggest displaying system options on smart-screens may be a viable method for overcoming difficulties of conveying VA options auditorily [10].

Similarly, we found very few differences in the types of tasks performed by partners in the same household. Additionally, many of the tasks our pairs performed with VAs have been identified as common in prior work examining users with no specified disability. For example, setting timers, controlling lights, checking weather, and accessing media are common for adult VA users $[4,10]$, homework help is a common use of VAs for children [28], and using VAs in household rituals, like playing games, is common for families [38].

However, observed similarities between partners with and without visual impairments do not imply visual abilities had no impact on VA adoption and use. PWVI in our sample often attributed their use of VAs directly to their visual (dis)abilities and wider ecosystems of inaccessibility-in kitchen appliances, thermostats, and even screen readerswhich make VAs the most, and often the only, accessible method for completing a task. Abdolrahmani et al. noted that a lack of accessible alternatives may contribute to PWVI remaining positive about VAs, despite their accessibility issues [1]. Our findings support this conclusion and suggest users with and without visual impairments likely hold contrasting views of VAs because of the relative advantage of using VAs for PWVI, over alternatives.

Because the relative advantage of using VAs is often higher for PWVI, in our sample, PWVI were frequently the household's primary VA user. PWVI demonstrated higher motivation to acquire, use, and learn about VAs than their sighted partners. For example, negotiations between family members have been identified as an important factor in understanding domestic technology adoption [23]. But, some PWVI in our sample were motivated enough to acquire a VA to sidestep negotiations with their partner entirely, like Ben, who ordered a VA without Carrie's (V) knowledge. After VAs were adopted, PWVI in our sample each reported using VAs as often as, or more often than, their sighted partners. Additionally, PWVI learned to use VAs more proactively than sighted partners, and disseminated their knowledge to their family. Ben sometimes beckoned Carrie (V) to teach her about VA features, calling "Hey, Babe! You got to check this out!" Similarly, despite Yvette's (V) apprehension of VAs, Hung demonstrated their use for her, and encouraged her to use
VAs to set timers, instead of continuing to use methods which are inaccessible to him. We observed many such cases where sighted family members learned how to use VAs primarily from more-knowledgeable PWVI, through direct instruction or mimicry. So, paradoxically, similarities in the types of tasks performed by partners with and without visual impairments in the same household may be due to differences in how PWVI and sighted partners learned to use VAs. It is possible household members used VAs similarly because each learned from PWVI.

Although many PWVI expressed pride in sharing their knowledge of VAs with their families, being the primary user of VAs can be a tenuous role in the home. Rode found previously that being the primary user of a technology can be advantageous for demonstrating knowledge to assert equality, but may also require assuming additional domestic labor [42]. Through this lens, PWVIs' proactivity in adopting and using VAs can be viewed as a domestic contribution and assertion of equality, and also as an added responsibility to maintain their own access amidst their families' activities. Inaccessibility in the home has been shown to exclude PWVI from contributing to the domestic labor of the household, disproportionately burdening sighted housemates and creating tensions in their interpersonal relationships [13]. We found many ways that VAs lowered access barriers, and enabled PWVI to participate in household tasks, like grocery shopping, supervising homework, and finding recipes for dinner. Some partners with vision, like Paula (V), noted that reducing inaccessibility in domestic labor for their partner has "taken a little work off" them personally. But, given the responsibility of being the primary VA user, it is uncertain whether adopting VAs into the home eased domestic burdens for PWVI. Perhaps most importantly, because VAs were often identified as the only accessible method for PWVI to accomplish some domestic task, it is not clear that the role - and associated work - of being the primary VA user was entirely optional for PWVI.

Collectively, our findings suggest that the (dis)abilities of one family member may change adoption and use patterns for an entire household. Dawe found similarly that AT adoption is impacted by the needs of non-disabled stakeholders within a family - not only those of the AT user [19]. We extend this conclusion, finding non-disabled partners' technology adoption and use were influenced by the desires of PWVI, and family (dis)abilities affected adoption and use, even though VAs are mainstream devices. Future work might further explore effects of access and (dis)ability on mainstream technology use in families.

\section{Universal Usability in the Home}

We found the universal usability of VAs may have advantages over accessibility strategies which provide access to either PWVI or sighted users, individually. For example, tactile markers on microwaves make it accessible to PWVI, but occlude the vision of sighted companions 
[13]. Similarly, to accommodate sighted coworkers' access to auditory information, PWVI often sacrifice their own access by turning off screen readers in meetings [14]. We did not observe universally usable VAs to impede the access of either partner by their presence. Rather, universal usability created risk-reward tradeoffs for families, which they weighed against increased access for PWVI.

One advantage of universal usability is that it presented new opportunities to for positive interpersonal interactions around and through a shared device for all family members, most notably for PWVI and their children. The unique accessibility of VAs has been previously identified for PWVI [1,15,40,53] and for children [21,27,28,35,59], separately. But, their universal usability for both PWVI and children created opportunities for positive mixed-visualability parent/child experiences. Notably, our interview script did not include direct questions about using VAs for parenting. Yet, discussions of parenting arose frequently and organically from other lines of inquiry. Storer and Branham found that inaccessibility in parenting is a significant issue for PWVI [49]. Our findings support this conclusion, and suggest VAs may be appropriate tools for supporting more accessible parent/child interactions.

Despite these benefits, the universal usability provided by VAs to PWVI and their children was not straightforward. Though the universal usability of VAs allowed PWVI and children to play together, it also created new anxieties about potential access to adult content for children. Universal usability has been previously explored as a design strategy for fostering collaboration primarily in teams of mixedability adults [37,57] and mixed-ability children [33,52]. Our findings suggest, in intergenerational mixed-ability teams, universal usability should be tempered by the recognition that age, like ability, presents different access needs, where it can be beneficial to restrict children's access. In contrast to concerns about children's access to VAs, universal usability for adult partners with different visual abilities was perceived positively in most situations. Several partners identified ways that universal usability led to natural collaborations on domestic tasks. For example, Ryan and Cooper (V) independently added items to a shared virtual shopping cart on their VA, to collaboratively maintain their stock of household essentials.

But, integrating universally usable VAs into the home often required partners to reach agreements about acceptable use of the shared device. Because the relative advantage of using VAs was much higher for PWVI, who may have no alternative, the risk/reward tradeoffs of integrating VAs into the home were uneven for partners. So, most often when agreements were reached either 1) PWVI were not able to use VAs for something they desired, because the risk was too great for their sighted partner, or 2) sighted partners used VAs for something they might not otherwise, because the reward was high enough for PWVI. For example, Fatima expressed interest in acquiring VA-connected home automation devices. But, her family did not own any, because Ahmed (V) perceived the risk of malicious actors as too large. Conversely, Ryan and Cooper (V) each made purchases using VAs, though they expressed concerns about having their credit card information stolen, because the advantage for Ryan of avoiding a rideshare was high enough for the pair to take a perceived risk.

Integrating novel access technologies into the home often requires cohabitants to reach agreements, like these, because individual access needs can conflict [13]. Though VAs did not create direct access conflicts, their universal usability did not guarantee equal access for all household members. Rather, the tradeoffs of owning VAs complicated partners' negotiations about use of a shared device, where the uneven benefits of VAs for partners often resulted in limitations placed on the access provided to PWVI. While universal usability is an important goal of designing for mixed-ability settings, focusing only on a technology's mutual accessibility may obfuscate concerns of owning a device which limit its practicality in context. Our findings of the complex social considerations PWVI weighed against access echo previous calls for designers to consider more holistic lived experiences of PWD ([13,30,47], for example), and not only their individual accessibility needs.

\section{LIMITATIONS AND FUTURE WORK}

A limitation of this study is its small sample size, which is common in accessibility research [20]. Our findings should not be interpreted as an exhaustive list of the tradeoffs of adopting VAs. They exemplify the complex negotiations involved in creating access in the home for PWD and their families. Additionally, like many studies of accessibility, used remote interview methods because of the difficulty of recruiting PWD [20,40]. While remote methods are pragmatic, many of our findings were contextualized by the family dynamics conveyed during our sessions. Conducting interviews with intimate-partner pairs allowed us more insight into participants' home lives than could be expected in individual interviews. But, there is space for work using in-home, ethnographic methods, where a researcher would gain deeper insight into participants' domestic contexts.

\section{CONCLUSION}

In this work we investigated the way that universally usable VAs are used in mixed-visual-ability homes. Although PWVI were more motivated to acquire VAs, used them more frequently, and learned more proactively about their features, partners with vision identified similar benefits and disadvantages of having VAs in their home. We found that the universal usability of VAs both equalizes experience across abilities and presents complex tradeoffs for families which are weighed against accessibility benefits for PWVI and complicate the decision to integrate VAs in the home.

\section{ACKNOWLEDGEMENTS}

The authors thank Sophie Barness for support in conducting this study, and Gillian Hayes, Dan Russell, and Mike Brzozowski for feedback on earlier versions of this work. 


\section{REFERENCES}

[1] Ali Abdolrahmani, Ravi Kuber, and Stacy M. Branham. 2018. "Siri Talks at You": An Empirical Investigation of Voice-Activated Personal Assistant (VAPA) Usage by Individuals Who Are Blind. In Proceedings of the 20th International ACM SIGACCESS Conference on Computers and Accessibility (ASSETS '18), 249-258. https://doi.org/10.1145/3234695.3236344

[2] James L. Alty and Dimitrios I. Rigas. 1998. Communicating Graphical Information to Blind Users Using Music: The Role of Context. In Proceedings of the SIGCHI Conference on Human Factors in Computing Systems (CHI '98), 574-581. https://doi.org/10.1145/274644.274721

[3] Morgan G. Ames, Janet Go, Joseph “Jofish" Kaye, and Mirjana Spasojevic. 2010. Making Love in the Network Closet: The Benefits and Work of Family Videochat. In Proceedings of the 2010 ACM Conference on Computer Supported Cooperative Work (CSCW '10), 145-154. https://doi.org/10.1145/1718918.1718946

[4] Tawfiq Ammari, Jofish Kaye, Janice Y. Tsai, and Frank Bentley. 2019. Music, Search, and IoT: How People (Really) Use Voice Assistants. ACM Trans. Comput.Hum. Interact. 26, 3: 17:1-17:28. https://doi.org/10.1145/3311956

[5] Shiri Azenkot and Nicole B. Lee. 2013. Exploring the Use of Speech Input by Blind People on Mobile Devices. In Proceedings of the 15th International ACM SIGACCESS Conference on Computers and Accessibility (ASSETS '13), 11:1-11:8. https://doi.org/10.1145/2513383.2513440

[6] Saminda Sundeepa Balasuriya, Laurianne Sitbon, Andrew A. Bayor, Maria Hoogstrate, and Margot Brereton. 2018. Use of Voice Activated Interfaces by People with Intellectual Disability. In Proceedings of the 30th Australian Conference on Computer-Human Interaction (OzCHI '18), 102-112. https://doi.org/10.1145/3292147.3292161

[7] Genevieve Bell, Mark Blythe, and Phoebe Sengers. 2005. Making by Making Strange: Defamiliarization and the Design of Domestic Technologies. ACM Trans. Comput.-Hum. Interact. 12, 2: 149-173. https://doi.org/10.1145/1067860.1067862

[8] Erin Beneteau, Olivia K. Richards, Mingrui Zhang, Julie A. Kientz, Jason Yip, and Alexis Hiniker. 2019. Communication Breakdowns Between Families and Alexa. In Proceedings of the 2019 CHI Conference on Human Factors in Computing Systems (CHI '19), 243:1-243:13. https://doi.org/10.1145/3290605.3300473

[9] Cynthia L Bennett, Erin Brady, and Stacy M Branham. 2018. Interdependence as a Frame for Assistive Technology Research and Design. In Proceedings of the 20th International ACM SIGACCESS Conference on Computers and Accessibility, 161-173.
[10] Frank Bentley, Chris Luvogt, Max Silverman, Rushani Wirasinghe, Brooke White, and Danielle Lottridge. 2018. Understanding the Long-Term Use of Smart Speaker Assistants. Proc. ACM Interact. Mob. Wearable Ubiquitous Technol. 2, 3: 91:1-91:24.

https://doi.org/10.1145/3264901

[11] Jeffrey P. Bigham, Ryan S. Kaminsky, Richard E. Ladner, Oscar M. Danielsson, and Gordon L. Hempton. 2006. WebInSight:: Making Web Images Accessible. In Proceedings of the 8th International ACM SIGACCESS Conference on Computers and Accessibility (Assets '06), 181-188. https://doi.org/10.1145/1168987.1169018

[12] Jeffrey P Bigham, Craig M Prince, and Richard E Ladner. 2008. WebAnywhere: a screen reader on-thego. In Proceedings of the 2008 international crossdisciplinary conference on Web accessibility (W4A), 7382.

[13] Stacy M Branham and Shaun K Kane. 2015. Collaborative accessibility: How blind and sighted companions co-create accessible home spaces. In Proceedings of the 33rd Annual ACM Conference on Human Factors in Computing Systems, 2373-2382.

[14] Stacy M Branham and Shaun K Kane. 2015. The invisible work of accessibility: how blind employees manage accessibility in mixed-ability workplaces. In Proceedings of the 17th International ACM SIGACCESS Conference on Computers \& Accessibility, 163-171.

[15] Stacy M. Branham and Antony Rishin Mukkath Roy. 2019. Reading Between the Guidelines: How Commercial Voice Assistant Guidelines Hinder Accessibility for Blind Users. In Proceedings of the ACM SIGACCESS Conference on Computers \& Accessibility (ASSETS '19).

[16] Minji Cho, Sang-su Lee, and Kun-Pyo Lee. 2019. Once a Kind Friend is Now a Thing: Understanding How Conversational Agents at Home Are Forgotten. In Proceedings of the 2019 on Designing Interactive Systems Conference (DIS '19), 1557-1569. https://doi.org/10.1145/3322276.3322332

[17] Eric Corbett and Astrid Weber. 2016. What Can I Say?: Addressing User Experience Challenges of a Mobile Voice User Interface for Accessibility. In Proceedings of the 18th International Conference on HumanComputer Interaction with Mobile Devices and Services (MobileHCI '16), 72-82. https://doi.org/10.1145/2935334.2935386

[18] Benjamin R. Cowan, Nadia Pantidi, David Coyle, Kellie Morrissey, Peter Clarke, Sara Al-Shehri, David Earley, and Natasha Bandeira. 2017. "What Can I Help You with?”: Infrequent Users' Experiences of Intelligent Personal Assistants. In Proceedings of the 19th International Conference on Human-Computer Interaction with Mobile Devices and Services 
(MobileHCI '17), 43:1-43:12. https://doi.org/10.1145/3098279.3098539

[19] Melissa Dawe. 2006. Desperately Seeking Simplicity: How Young Adults with Cognitive Disabilities and Their Families Adopt Assistive Technologies. In Proceedings of the SIGCHI Conference on Human Factors in Computing Systems (CHI '06), 1143-1152. https://doi.org/10.1145/1124772.1124943

[20] Marianne Dee and Vicki L. Hanson. 2016. A Pool of Representative Users for Accessibility Research: Seeing Through the Eyes of the Users. ACM Trans. Access. Comput. 8, 1: 4:1-4:31. https://doi.org/10.1145/2845088

[21] Daniel Fitton, Janet C Read, Gavin Sim, and Brendan Cassidy. 2018. Co-designing Voice User Interfaces with Teenagers in the Context of Smart Homes. In Proceedings of the 17th ACM Conference on Interaction Design and Children (IDC '18), 55-66. https://doi.org/10.1145/3202185.3202744

[22] Richard Guy and Khai Truong. 2012. CrossingGuard: exploring information content in navigation aids for visually impaired pedestrians. In Proceedings of the SIGCHI Conference on Human Factors in Computing Systems, 405-414.

[23] Debby Hindus. 1999. The Importance of Homes in Technology Research. In Cooperative Buildings. Integrating Information, Organizations, and Architecture (Lecture Notes in Computer Science), 199207.

[24] Tejinder K. Judge, Carman Neustaedter, and Andrew F. Kurtz. 2010. The family window: the design and evaluation of a domestic media space. 2361-2370. https://doi.org/10.1145/1753326.1753682

[25] Shaun K. Kane, Jeffrey P. Bigham, and Jacob O. Wobbrock. 2008. Slide Rule: Making Mobile Touch Screens Accessible to Blind People Using Multi-touch Interaction Techniques. In Proceedings of the 10th International ACM SIGACCESS Conference on Computers and Accessibility (Assets '08), 73-80. https://doi.org/10.1145/1414471.1414487

[26] Steven Kelley. 2016. Google Home: A New Electronic Digital Assistant - VisionAware. VisionAware. Retrieved September 2, 2019 from https://www.visionaware.org/info/everydayliving/helpful-products/using-a-computer/googlehome $/ 1245$

[27] Silvia B. Lovato, Anne Marie Piper, and Ellen A. Wartella. 2019. Hey Google, Do Unicorns Exist?: Conversational Agents As a Path to Answers to Children's Questions. In Proceedings of the 18th ACM International Conference on Interaction Design and Children (IDC '19), 301-313. https://doi.org/10.1145/3311927.3323150

[28] Silvia Lovato and Anne Marie Piper. 2015. "Siri, is This You?”: Understanding Young Children's Interactions with Voice Input Systems. In Proceedings of the 14th International Conference on Interaction Design and Children (IDC'15), 335-338. https://doi.org/10.1145/2771839.2771910

[29] Ewa Luger and Abigail Sellen. 2016. "Like Having a Really Bad PA": The Gulf Between User Expectation and Experience of Conversational Agents. In Proceedings of the 2016 CHI Conference on Human Factors in Computing Systems (CHI '16), 5286-5297. https://doi.org/10.1145/2858036.2858288

[30] Jennifer Mankoff, Gillian R. Hayes, and Devva Kasnitz. 2010. Disability studies as a source of critical inquiry for the field of assistive technology. In Proceedings of the 12th international ACM SIGACCESS conference on Computers and accessibility, 3-10.

[31] Melissa Mazmanian and Simone Lanette. 2017. "Okay, One More Episode": An Ethnography of Parenting in the Digital Age. In Proceedings of the 2017 ACM Conference on Computer Supported Cooperative Work and Social Computing (CSCW '17), 2273-2286. https://doi.org/10.1145/2998181.2998218

[32] J.J. Meddaugh. 2017. There's No Place like Google Home: A Review of Google's Voice Assistant | AccessWorld $\mid$ American Foundation for the Blind. American Foundation for the Blind. Retrieved September 2, 2019 from https://www.afb.org/aw/18/3/15230

[33] Oussama Metatla, Alison Oldfield, Taimur Ahmed, Antonis Vafeas, and Sunny Miglani. 2019. Voice User Interfaces in Schools: Co-designing for Inclusion with Visually-Impaired and Sighted Pupils. In Proceedings of the 2019 CHI Conference on Human Factors in Computing Systems (CHI '19), 378:1-378:15. https://doi.org/10.1145/3290605.3300608

[34] Chelsea Myers, Anushay Furqan, Jessica Nebolsky, Karina Caro, and Jichen Zhu. 2018. Patterns for How Users Overcome Obstacles in Voice User Interfaces. In Proceedings of the 2018 CHI Conference on Human Factors in Computing Systems (CHI '18), 6:1-6:7. https://doi.org/10.1145/3173574.3173580

[35] Luiza Superti Pantoja, Kyle Diederich, Liam Crawford, and Juan Pablo Hourcade. 2019. Voice Agents Supporting High-Quality Social Play. In Proceedings of the 18th ACM International Conference on Interaction Design and Children (IDC '19), 314-325. https://doi.org/10.1145/3311927.3323151

[36] Victoria Petrock. US Voice Assistant Users 2019. eMarketer. Retrieved September 17, 2019 from https://www.emarketer.com/content/us-voice-assistantusers-2019

[37] Anne Marie Piper and James D Hollan. 2008. Supporting medical conversations between deaf and hearing individuals with tabletop displays. In 
Proceedings of the 2008 ACM conference on Computer supported cooperative work, 147-156.

[38] Martin Porcheron, Joel E. Fischer, Stuart Reeves, and Sarah Sharples. 2018. Voice Interfaces in Everyday Life. In Proceedings of the 2018 CHI Conference on Human Factors in Computing Systems (CHI '18), 640:1-640:12. https://doi.org/10.1145/3173574.3174214

[39] François Portet, Michel Vacher, Caroline Golanski, Camille Roux, and Brigitte Meillon. 2013. Design and Evaluation of a Smart Home Voice Interface for the Elderly: Acceptability and Objection Aspects. Personal Ubiquitous Comput. 17, 1: 127-144. https://doi.org/10.1007/s00779-011-0470-5

[40] Alisha Pradhan, Kanika Mehta, and Leah Findlater. 2018. "Accessibility Came by Accident": Use of VoiceControlled Intelligent Personal Assistants by People with Disabilities. In Proceedings of the 2018 CHI Conference on Human Factors in Computing Systems (CHI '18), 459:1-459:13. https://doi.org/10.1145/3173574.3174033

[41] Amanda Purington, Jessie G. Taft, Shruti Sannon, Natalya N. Bazarova, and Samuel Hardman Taylor. 2017. "Alexa is My New BFF": Social Roles, User Satisfaction, and Personification of the Amazon Echo. In Proceedings of the 2017 CHI Conference Extended Abstracts on Human Factors in Computing Systems (CHI EA '17), 2853-2859. https://doi.org/10.1145/3027063.3053246

[42] Jennifer A. Rode. 2010. The Roles That Make the Domestic Work. In Proceedings of the 2010 ACM Conference on Computer Supported Cooperative Work (CSCW '10), 381-390. https://doi.org/10.1145/1718918.1718984

[43] David A. Ross and Bruce B. Blasch. 2000. Wearable interfaces for orientation and wayfinding. In Proceedings of the fourth international ACM conference on Assistive technologies, 193-200.

[44] S. Schlögl, G. Chollet, M. Garschall, M. Tscheligi, and G. Legouverneur. 2013. Exploring Voice User Interfaces for Seniors. In Proceedings of the 6th International Conference on PErvasive Technologies Related to Assistive Environments (PETRA '13), 52:152:2. https://doi.org/10.1145/2504335.2504391

[45] Alex Sciuto, Arnita Saini, Jodi Forlizzi, and Jason I. Hong. 2018. "Hey Alexa, What's Up?": A MixedMethods Studies of In-Home Conversational Agent Usage. In Proceedings of the 2018 Designing Interactive Systems Conference (DIS '18), 857-868. https://doi.org/10.1145/3196709.3196772

[46] Kristen Shinohara, Cynthia L. Bennett, and Jacob O. Wobbrock. 2016. How Designing for People With and Without Disabilities Shapes Student Design Thinking. In Proceedings of the 18th International ACM
SIGACCESS Conference on Computers and Accessibility (ASSETS '16), 229-237. https://doi.org/10.1145/2982142.2982158

[47] Kristen Shinohara, Jacob O. Wobbrock, and Wanda Pratt. 2018. Incorporating Social Factors in Accessible Design. In Proceedings of the 20th International ACM SIGACCESS Conference on Computers and Accessibility (ASSETS '18), 149-160. https://doi.org/10.1145/3234695.3236346

[48] Ben Shneiderman. 2000. Universal usability. Communications of the ACM 43, 5: 85-85.

[49] Kevin M. Storer and Stacy M. Branham. 2019. “ That's the Way Sighted People Do It": What Blind Parents Can Teach Technology Designers About Co-Reading with Children. In Proceedings of the 2019 on Designing Interactive Systems Conference, 385-398.

[50] Alexander Thayer, Matthew J. Bietz, Katie Derthick, and Charlotte P. Lee. 2012. I Love You, Let's Share Calendars: Calendar Sharing As Relationship Work. In Proceedings of the ACM 2012 Conference on Computer Supported Cooperative Work (CSCW '12), 749-758. https://doi.org/10.1145/2145204.2145317

[51] Anja Thieme, Cynthia L Bennett, Cecily Morrison, Edward Cutrell, and Alex S Taylor. 2018. I can do everything but see!-How People with Vision Impairments Negotiate their Abilities in Social Contexts. In Proceedings of the 2018 CHI Conference on Human Factors in Computing Systems, 203.

[52] Anja Thieme, Cecily Morrison, Nicolas Villar, Martin Grayson, and Siân Lindley. 2017. Enabling Collaboration in Learning Computer Programing Inclusive of Children with Vision Impairments. In Proceedings of the 2017 Conference on Designing Interactive Systems (DIS '17), 739-752. https://doi.org/10.1145/3064663.3064689

[53] Alexandra Vtyurina, Adam Fourney, Meredith Ringel Morris, Leah Findlater, and Ryen W. White. 2019. Bridging Screen Readers and Voice Assistants for Enhanced Eyes-Free Web Search. In The World Wide Web Conference (WWW '19), 3590-3594. https://doi.org/10.1145/3308558.3314136

[54] Emily Q. Wang and Anne Marie Piper. 2018. Accessibility in Action: Co-Located Collaboration Among Deaf and Hearing Professionals. Proc. ACM Hum.-Comput. Interact. 2, CSCW: 180:1-180:25. https://doi.org/10.1145/3274449

[55] Brian Wentz, Paul T. Jaeger, and Jonathan Lazar. 2011. Retrofitting accessibility: The legal inequality of afterthe-fact online access for persons with disabilities in the United States. First Monday 16, 11. https://doi.org/10.5210/fm.v16i11.3666

[56] Michele A Williams, Caroline Galbraith, Shaun K Kane, and Amy Hurst. 2014. Just let the cane hit it: how the blind and sighted see navigation differently. In 
Proceedings of the 16th international ACM SIGACCESS conference on Computers \& accessibility, 217-224.

[57] Fredrik Winberg and John Bowers. 2004. Assembling the Senses: Towards the Design of Cooperative Interfaces for Visually Impaired Users. In Proceedings of the 2004 ACM Conference on Computer Supported Cooperative Work (CSCW '04), 332-341. https://doi.org/10.1145/1031607.1031662

[58] Rayoung Yang, Sangmi Park, Sonali R. Mishra, Zhenan Hong, Clint Newsom, Hyeon Joo, Erik Hofer, and Mark W. Newman. 2011. Supporting spatial awareness and independent wayfinding for pedestrians with visual impairments. In The proceedings of the 13th international ACM SIGACCESS conference on Computers and accessibility, 27-34.

[59] Svetlana Yarosh, Stryker Thompson, Kathleen Watson, Alice Chase, Ashwin Senthilkumar, Ye Yuan, and A. J. Bernheim Brush. 2018. Children Asking Questions: Speech Interface Reformulations and Personification Preferences. In Proceedings of the 17th ACM Conference on Interaction Design and Children (IDC '18), 300-312. https://doi.org/10.1145/3202185.3202207
[60] Hanlu Ye, Meethu Malu, Uran Oh, and Leah Findlater. 2014. Current and Future Mobile and Wearable Device Use by People with Visual Impairments. In Proceedings of the SIGCHI Conference on Human Factors in Computing Systems (CHI '14), 3123-3132. https://doi.org/10.1145/2556288.2557085

[61] Chien Wen Yuan, Benjamin V. Hanrahan, Sooyeon Lee, Mary Beth Rosson, and John M. Carroll. 2017. I Didn't Know That You Knew I Knew: Collaborative Shopping Practices Between People with Visual Impairment and People with Vision. Proc. ACM Hum.-Comput. Interact. 1, CSCW: 118:1-118:18. https://doi.org/10.1145/3134753

[62] JAWS ${ }^{\circledR}$ - Freedom Scientific. Retrieved September 10, 2019 from https://www.freedomscientific.com/products/software/ja ws/

[63] NV Access. Retrieved September 10, 2019 from https://www.nvaccess.org/

[64] Talking Products. Retrieved September 10, 2019 from https://assistech.com/store/talking-products 